\title{
Suchtberatung im Strafvollzug
}

\section{"Bewusstein lässt sich nicht einsperren" - Erfahrungsbericht eines Suchtberaters \\ Jürgen Oetting}

\section{Einführung}

$\mathrm{D}$ ie Ausgangslage, die ein Suchtberater im Strafvollzug vorfindet, ist alles andere als rosig. Meine Stammkunden haben ihre Chancen schon verpasst. Sie sind Anfang bis Mitte 30, geschieden oder getrennt, haben durchschnittlich zwei Kinder, die entweder bei der leiblichen Mutter, in Pflegefamilien oder bei Adoptiveltern leben. Sie haben die Sonder- oder Hauptschule ohne Abschluss verlassen und eine Berufsausbildung abgebrochen oder nie begonnen. Sie sind seit mehreren Jahren arbeitslos. Sie haben Alkoholprobleme, begingen mehrere Straftaten und verbüssen ihre dritte oder vierte Freiheitsstrafe.

Ich arbeite als externer (aber täglich anwesender) Suchtberater in der JVA Kiel und bin für den Bereich „legale Drogen“ zuständig. Für die Konsumenten illegaler Drogen sind andere Berater da, eine Trennung die weniger dem wirklichen Leben als den deutschen Strafgesetzen entspricht. Die JVA Kiel ist eine Anstalt des Regelvollzugs, für Männer, die als Wiederholungstäter gelten und jeweils nicht länger als drei Jahre einsitzen müssen. Ausser denjenigen, die eine Ersatzfreiheitsstrafe verbüssen, haben alle Kieler Gefangenen bereits Knasterfahrung. Dort also war ich im Sommer 2006 seit fünf Jahren tätig, Anlass für einen Erfahrungsbericht und einen skeptischen Ausblick.

\section{Wege von Süchtigen - „Teufelskreis"}

Wenn die Gefangenen während ihrer Haft Suchthilfe beanspruchen und eine Alkoholentwöhnungsbehandlung anstreben, wenn die ihnen vom zuständigen Kostenträger auch finanziert wird und ein Therapieplatz organisiert ist, werden sie im Normalfall nach zwei Dritteln ihrer Haftzeit auf Bewährung entlassen (§ 57 StGB), wobei dann die Teilnahme an der Entwöhnungsbehandlung meist eine der Bewährungsauflagen ist. Wenn ein so Entlassener dann auch tatsächlich in der Fachklinik ankommt (was längst nicht immer der Fall ist) und dort acht bis 16 Wochen (die derzeitige Dauer von stationären Standardbehandlungen) an seinem Problem arbeitet und arbeiten lässt, wird er alkoholabstinent in seine alte Wohngegend entlassen. Oder man hat ihn in der Fachklinik davon überzeugen können, dass ein Wechsel des sozialen Umfeldes sinnvoll ist. Nur wird die neue Wohngegend so anders nicht sein können, grosse Wahlmöglichkeiten haben ja haftentlassene Hartz-IV-Empfänger nicht.

Da sitzt er dann also „trocken“ in einer kleinen „preiswerten“ Mietwohnung am Ortsrand oder in einem heruntergekommenen Innenstadtviertel, ohne Arbeit, ohne Familie, meist ohne Hobby - ohne Bindung und ohne Anerkennung. Mit viel Willenskraft hangelt er sich anfangs eventuell von den Nachsorgeterminen in der örtlichen Suchtberatungstelle zu den Treffen seiner Selbsthilfegruppe. Da kann Bindung entstehen und Anerkennung gibt es auch. Aber ob das reicht? Diesem Klienten (und den vielen anderen „Stammkunden”) offenbar nicht, irgendwann steht er wieder zwischen alten und neuen Saufkumpanen im Park oder vor dem Supermarkt. Und irgendwann sehe ich ihn dann wieder - im Knast. Und nach ein paar Monaten Haft wird dann die nächste Therapie beantragt.

Das nervt, weil es so sinnlos wirkt. Aber bevor es nervt, muss man einige Jahre im Gefängnis arbeiten, denn es braucht seine Zeit, bevor die alten Bekannten wieder eintrudeln. Nach fünf Jahren sind nun einige schon zum dritten Mal wieder da. Altgediente Beamte des Allgemeinen Vollzugsdienstes verfügen über einen noch grösseren Erfahrungsschatz und begrüssen manchen Gefangenen schon zum sechsten oder siebten Mal - und können sich ein abfälliges Grinsen kaum verkneifen, wenn solche Gefangene dann wieder einen Antrag auf Suchtberatung stellen. Der Weg eines Inhaftierten über die Suchthilfe nach
Hause und kurz darauf zurück in den Vollzug ist damit als ein „Teufelskreis“ vorgezeichnet.

\section{Die „Stammkunden”}

Diese „Stammkunden” bilden eine kräftige Minderheit in der statistischen Gesamtmenge meiner Klienten, sind aber keinesfalls repräsentativ für den Regelvollzug, für den Erwachsenen-Erstvollzug und für den Jugendstrafvollzug schon gar nicht. Aber sie binden viel Beratungs- und Vermittlungszeit und kommen mir deshalb „typisch” vor. Diejenigen, die von mir nur einmal in eine Entwöhnungsbehandlung oder an eine ambulante Beratungsstelle vermittelt wurden, verschwinden mit der Haftentlassung aus meinem Fokus und verteilen sich auf das gesamte Bundesland. Die Erfolge meiner Arbeit werden somit kaum registriert. Längerfristige Therapieerfolge sehe ich also nie, kann sie nur vermuten. Rückfälle in Alkoholabhängigkeit und Straffälligkeit dagegen werden mir mit den altbekannten Neuzugängen präsentiert. Und diese altbekannten Klienten sind unter den Gefangenen Multiplikatoren, vermitteln ihre „Therapieerfahrungen" in der Anstalt weiter. Bei manchen von ihnen entwickelt sich von Haft zu Haft ein "gefühlter Therapieanspruch" sobald der Zweidritteltermin in Sicht kommt. Dann wird die Anbahnung einer stationären Entwöhnungsbehandlung ohne grosse Umschweife von mir gefordert: „Ich brauch' 'ne Therapie, bald ist Zweidritteltermin - zur Anhörung muss ich die Kostenzusage haben." Ein Gefangener ist sogar (erfolglos) juristisch gegen mich vorgegangen, weil ich in meiner Sozialberichts-Stellungnahme Zweifel an seiner Behandlungsmotivation geäussert hatte, ihm also die gewünschte Kosten-

Mit viel Aufwand wird nach der Föderalismusreform statt des Vorrangs der Resozialisierung nur noch von Chancenvollzug geredet. Neue Formeln halten Einzug in die Ländervollzugsgesetze. Aber die Probleme bleiben: etwa die ,Kultur' der Widerspenstigkeit, die sich weder mit Resozialisierung noch Chancenvollzug und schon gar nicht mit noch mehr Strafrecht beseitigen lässt. Hier wären bisweilen Kosten sparende und humane Angebote nützlicher als immer wieder neue Slogans. Wieso ist Realismus so schwer und kriminalpolitische Formeln so beliebt? 
zusage nicht organisiert hatte. Er hatte bereits vier stationäre Entwöhnungsbehandlungen hinter sich - das Leben in der Suchtberatung kann sehr deprimierend sein.

\section{Worum es eigentlich geht - Haftlockerungen}

Ähnlich verhält es sich mit zweckgebundenen Ausgängen in Selbsthilfegruppen. Manche Klienten nehmen drei oder vier Beratungstermine wahr, um dann mit dem wahren Anliegen herauszurücken: „Nun könnte ich doch auch externe Selbsthilfegruppen besuchen, können Sie dazu bitte 'ne Stellungsnahme schreiben." Oft genug tue ich das dann sogar, denn gegen den Besuch einer Selbsthilfegruppe spricht aus suchtberaterischer Sicht wenig - und über die Haftlockerung habe ja nicht ich zu befinden. Um die aber geht es fast immer (auch). Ausserdem ist der Besuch von Selbsthilfegruppen kostenlos - eine stationäre Entwöhnungsbehandlung kostet dagegen die Öffentliche Hand schnell mal 10.000 Euro.

\section{Die unterschiedliche Behandlung von Drogen- und Alkoholabhängigen}

$\mathrm{Zu}$ Beginn meiner Arbeit als GefängnisSuchtberater irritierten mich andere Beobachtungen. Besonders ärgerlich fand ich (und zwar schon während meiner Tätigkeit in einer öffentlichen Beratungsstelle) die völlige Schieflage in der allgemeinen Wahrnehmung: die Verteufelung jedweden Drogenkonsums und die Gleichgültigkeit gegenüber der Alkoholproblematik. Dabei sind Alkoholstraftaten (Wirkungskriminalität) deutlich schwerwiegender als die meisten Drogenstraftaten (Beschaffungskriminalität). Den Konsumenten illegaler Drogen galt zwei Jahrzehnte lang ein hohes mediales und wissenschaftliches Interesse. Diverse Forschungsprojekte wurden aufgelegt und das Drogenthema war immer für einen Zeitungs- oder Zeitschriftenartikel gut. Über missbräuchlichen Alkoholkonsum las man dagegen wenig. Das änderte sich erst vor wenigen Jahren, als nicht mehr zu übersehen war, dass Jugendliche massenhaft ein Hobby namens „Komasaufen“ pflegen (viele frühere „Komasäufer” sind übrigens inzwischen im Erwachsenenstrafvollzug angekommen).

Auch im Strafvollzug führt der (vom Gesetzgeber so gewollte) ganz unterschiedliche Umgang mit Konsumenten illegaler beziehungsweise Konsumenten legaler Drogen zu Irritationen - besonders unter den Gefangenen. Viele kommen im Glauben zu mir, sie könnten durch eine Entwöhnungs- behandlung das Gefängnis mit einer Klinik tauschen. Das ist aber für „Alkis” nicht möglich, nur für „Drogis”. Alkoholabhängige, deren Straftat erkennbar mit dem Trinken zusammenhängt, können allenfalls nach Zweidritteln (sehr selten auch zur Hälfte) ihrer Haftzeit entlassen werden und in eine Therapie gehen. Die $\$ \S 35$ ff. BtMG jedenfalls gelten nur für Drogenabhängige.

Auch unter den erfahrenen Knackis des Regelvollzuges hat sich noch nicht ganz herumgesprochen, was es mit „Therapie statt Strafe" auf sich hat. Manche haben, wenn sie von der „Bevorzugung“ der Drogenabhängigen hören und ihre Felle davon schwimmen sehen, die nahe liegende Idee: „Dann werde ich eben drogenabhängig." Das scheint in keinem Gefängnis ein grösseres Problem zu sein. Aber: „Therapie statt Strafe" gibt es trotzdem nicht. Da müsste dann die „Drogenabhängigkeit" schon im Zusammenhang mit dem Delikt, das dem Freiheitsentzug zugrunde liegt, stehen und dem Gericht bekannt gewesen sein.

\section{Angleichungstendenzen - Schaffung einer $\S \S 35$ ff BtMG vergleichbaren Norm}

Diese Zusammenhänge sind für meine inhaftierten Klienten (Standardspruch: „Die Drogenabhängigen kriegen hier alles, ich nichts.") und auch für mich nicht leicht zu akzeptieren, wenn doch jede Sucht eine Krankheit sein soll. Der vorigen Bundesregierung muss dieser Widerspruch ebenfalls aufgefallen sein. Im Auftrage des Bundesministeriums für Justiz wurde seit Dezember 2001 an der Kriminologischen Zentralstelle (KrimZ) in Wiesbaden der Frage nachgegangen, ob für alkoholabhängige Straftäter eine den $\S \S 35$ ff. BtMG entsprechende Regelung (Zurückstellung der Strafvollstreckung bei Durchführung einer Therapie) geschaffen werden sollte (http://www.krimz.de/dr-alkohol.html).

Zustimmung fanden diese Tendenzen in der Praxis. In mehreren Bundesländern wurden in Jusizvollzugsanstalten Daten erhoben. Bei der Staatsanwaltschaft beim Landgericht Mainz wurde eine Aktenanalyse durchgeführt und repräsentativ ausgewählte Richter, Staatsanwälte und Anstaltsleiter wurden schriftlich zu mutmasslichen (Neben-)Folgen einer den §§ 35 ff. BtMG vergleichbaren Regelung für alkoholabhängige Straftäter sowie zum praktischen Anwendungsbereich und möglichen Alternativen zu einer solchen Lösung befragt Ausserdem gab es im Rahmen des Forschungsprojektes im Februar 2002 eine zweitägige Expertenanhörung, wobei die meisten Fachleute die Auffassung vertraten, dass durch die Einführung einer entsprechenden Regelung für alkoholabhängige Täter das bestehende System sinnvoll ergänzt werden könne (http://www. krimz.de/dr-experten.html).

Die Studie ist längst abgeschlossen - ein Ergebnis gibt es noch nichtt. Eine Veröffentlichung des Ergebnisses war auf den Internetseiten der KrimZ ursprünglich für das Jahr 2005 angekündigt, inzwischen ist daraus das Jahr 2006 geworden. Bis zum Redaktionsschluss dieses Textes (31. August 2006) war auf den KrimZ-Webseiten davon nichts zu sehen. Auf meine Mailanfragen hiess es (einmal 2005, einmal 2006), das Ergebnis sei vom Bundesministerium für Justiz noch nicht freigegeben worden. Es passt wohl nicht in die kriminalpolitischen Stimmung dieser Jahre, denn eine analoge Anwendung des Prinzips „Therapie statt Strafe“ für Alkoholabhängige hiesse praktisch: Mehr Haftverschonung.

Haftverschonung oder Haftverkürzung das ist immer auch mitschwingendes wenn nicht sogar auslösende - Thema bei der Beratung und Therapievermittlung von Alkoholabhängigen im Regelvollzug. Nach meinen Erfahrungen geht es den wenigsten Klienten in erster Linie um die Auseinandersetzung mit ihrem Suchtproblem. Es geht immer auch darum, die Haftzeit zu verkürzen. Und in vielen Fällen geht es ausschliesslich darum. Am offenkundigsten wird das, wenn Klienten mir (oder ich ihnen) die „Knacki-Rechnung” aufmachen, zum Beispiel: Wenn jemand eine Freiheitsstrafe von 12 Monaten zu verbüssen hat, könnte er nach acht Monaten zum Zweidritteltermin entlassen werden und eine stationäre Entwöhnungsbehandlung beginnen. Die dauert (je nach Klinik und Kostenträger) acht bis sechzehn Wochen. Zweidrittelhaft und Therapiedauer entsprechen in diesem Fall zeitlich ziemlich genau der Vollverbüssung. Nach dieser „Rechnung" habe ich schon oft gehört: „Das bringt ja nichts, dann mache ich zentral." Was heisst, dann verbüsse ich voll - denn dann gibt es ja nicht einmal Bewährung.

\section{Auswirkungen einer Gleichbehandlung von Drogen- und Alkoholabhängigen}

Aus diesem Blickwinkel „rechnen” sich stationäre Alkoholentwöhnungsbehandlungen erst ab Haftstrafen, die deutlich länger sind als ein Jahr. Würde nun die Möglichkeit „Therapie statt Strafe” aus dem BtMG analog auch auf alkoholabhängige Strafgefangene angewandt, lohnte sich die Sache vom ersten Hafttag an. Und unter solchen Bedingungen gäbe es plötzlich deutlich 
mehr selbsterklärte Alkoholabhängige in den Gefängnissen. Zur Zeit wird von Inhaftierten eine manifeste Sucht häufig abgestritten - manchmal zu recht. Denn nicht jede Straftat unter Alkoholeinfluss ist Ausdruck einer Alkoholproblematik oder gar einer Alkoholabhängigkeit. Gerade Männer mit einer relativ geringen Alkoholtoleranz „untrainierte Trinker” also - können berauscht-enthemmt Straftaten begehen, die nüchtern nicht passiert wären. Für den Vollzug sind gerade solche Täter schwer einzuschätzen. Eine Alkoholentwöhnung ist dann jedenfalls nicht die angemessene Problemlösung.

Derartige Differenzierungen wären bei analoger Anwendung von "Therapie statt Strafe" perdu. Wie bei den inhaftierten Drogenkonsumenten würde sich jeder Alkoholkonsument gerne zum Süchtigen machen, wenn dadurch Haft verkürzt oder verhindert werden kann. Und Suchtberater würden damit zu informellen Haftverhinderern oder -verkürzern. Eine derart „sekundäre Funktion" mag ja im Feld der illegalen Drogen noch (klammheimlich) Sinn machen, denn Drogenkriminalität ist so ziemlich der letzte Bereich, auf den sich der (ansonsten konstruktivistisch einäugige) Labeling Approach anwenden lässt. Bei den AlkoholGewalttätern, -Verkehrsgefährdern und Vergewaltigern erschliesst sich mir ein derart abolotionistisches Hintergrundmotiv von Suchtberatung nicht. Und bei den anderen „Etikettierten“, den meist älteren, wohnungslosen chronisch alkoholabhängigen „Eierdieben“, denen ich gerne jeden Tag im Gefängnis ersparen würde, hilft keine Therapie mehr - für sie bleiben nur Betreuungseinrichtungen.

\section{Neue Wege - „Chancenvollzug”}

Doch die analoge Anwendung der §§ 35 ff. wird nicht kommen - jedenfalls nicht in den nächsten Jahren. Dafür aber der „Chancenvollzug". Von dem ist in den Vorbereitungspapieren für neue (Länder-)Strafvollzugsgesetze viel die Rede, ganz besonders wenn sie für CDU-Justizminister geschrieben sind. So heisst es beispielsweise in den „Eckpunkten für ein Niedersächsisches Strafvollzugsgesetz" vom Mai 2006, dass der Chancenvollzug gesetzlich verankert und zum zentralen Gestaltungsgrundsatz der Vollzugsplanung werden soll. Als mitarbeitsbereit geltende Gefangene erhalten danach die Chance zur Teilnahme am Chancenvollzug (Förderung, Therapie). Und nichtmitarbeitsbereite Gefangene werden danach nur im Rahmen einer "Grundversorgung" betreut. Selbstverständlich soll auch nichtmitarbeitsbereiten Gefangenen die Teilnahme am Chancenvollzug ermöglicht werden, sobald sie zu mitarbeitsbereiten Gefangenen mutiert sind.

Und dann! Dann gibt es Haftlockerung für diejenigen, die den Chancenvollzug genutzt haben. Im Grunde nichts Neues aus Hannover also. Wer das „Therapiespiel” mitspielen will und kann, wird mit Lockerung belohnt. Da werden dann die Gefangenen, so sie mitspielen wollen, eben noch mehr „Therapeutenschappi” an Psychologen, Sozialarbeiter und Vollzugsabteilungsleiter verfüttern. „Therapeutenschappi”, das ist im Knast- und Psychiatriejargon das, was die Helfer hören wollen. Und ob es sich bei der verbalen Produktion von Therapeutenfutter dann um ein wirkliches Einlassen auf begleitete Veränderungsprozesse geht oder eine Inszenierung zum Zwecke der Haftverkürzung, wer will das entscheiden können? Manche Gefangene des Regelvollzugs (manchmal auch noch mit Massregelvollzugsvergangenheit und sehr viel Training aus Kinder- und Jugendheimen) haben mehr Therapieerfahrung als ihre professionellen Helfer.

In den niedersächsischen Eckpunkten heisst es: „Uns geht es nicht um ein äusserliches, angepasstes Wohlverhalten, sondern um die Entscheidung des Gefangenen, ernsthaft an der Erreichung gemeinsam definierter Vollzugsziele mitzuarbeiten." $\mathrm{Zu}$ diesem Thema gibt es kritische Äußerungen. Der soziologische Systemtheoretiker Peter Fuchs hat in eimen legendären Vortrag (http://www.ibs-networld.de/altesferkel/sept-fuchs.shtml) vor Vollzugsexperten Ernüchterndes gesagt: schliessend „guten Gewissens" den reinen Verwahrvollzug zu installieren.

Peter Fuchs hat die Klemme, in der professionelle Helfer stecken, in seinem Vortrag benannt: Auch im Hinblick auf den Justizvollzug hätten sich Expertenkulturen entwickelt, „die sich auf die Organisation unorganisierbaren Bewusstseins beziehen, Kulturen, die aus Psychologie, Sozialarbeit, Sozialpädagogik ihr Personal rekrutieren. Da das Problem des widerspenstigen Bewusstseins aber nicht gelöst werden kann, wird stattdessen (und zum Vorteil für die damit beschäftigte Branche der Inklusionsingenieure) unabschliessbare Kommunikation erreicht, die die Fortführung des Systems garantiert. Zugleich wird damit eine Dauerkonfliktmöglichkeit zwischen den Expertenkulturen geschaffen, die (Re)Inklusion inszenieren wollen, und denjenigen, die die Exklusion (Separation, Allokation etc.) vollziehen müssen." - Oder es politisch wollen, müsste man heutzutage hinzufügen.

Der Fuchs-Text ist auf der Originalwebsite mit einer sich bewegenden Comicfigur in einer Zwangsjacke illustriert, die sich vergeblich müht, aus ihrer misslichen Situation heraus zu kommen. Nicht ganz so gefesselt, aber doch arg eingeengt von sich widersprechenden Motiven, Notwendigkeiten und Zwängen komme ich mir als Suchtberater im Strafvollzug häufig vor. Aber lieber so als in einem Verwahrvollzug, in dem alles eindeutig ist, aber nichts mehr geht.

Jürgen Oetting ist Suchtberater in der JVA Kiel.
„Das Bewusstsein ist - anders als die Körper, die es irgendwie bewohnt - schlechthin nicht einsperrbar. Und es liegt auf der Hand, dass es kaum davon zu überzeugen ist, dass es gleichsam mit seinem Körper irgendwohin verlegt und abgelegt werden soll. Man kann den Körper irgendwohin befördern, aber darf nicht erwarten, dass das Bewusstsein dies begeistert zur Kenntnis nimmt, dass es mitbefördert wird, (...) Die Folge ist zwingend, dass es der Justizvollzug in dieser Form immer mit einer ,Kultur' der Widerspenstigkeit zu tun hat, mit der Ausprägung eines informalen Mediums hartnäckiger Illegalität, mit der Entwicklung und dem Ausbau informaler Hierarchien und der Begünstigung jeder Strategie, die sich gegen das Ansinnen, das Bewusstsein im Vollzug gleichsam mitzuorganisieren, wehrt. (...) Verwunderung könnte dann dem Umstand gelten, warum sich irgendjemand darüber wundert oder gar die Auffassung vertritt (und entsprechende Formen zu schaffen sucht), dass das widerspenstige Bewusstsein, das gar nicht vermeidbar ist, diszipliniert werden müsse. Warum ist das Selbstverständliche ein Problem?"

Beziehungsweise: Weshalb wird das Unmögliche und Unmöglich-zu-prüfende zum Programm erhoben? Vielleicht, um ein krachendes Scheitern des Chancen-Behandlungsvollzuges $\mathrm{zu}$ provozieren $\mathrm{um}$ an- 